PROCEEDINGS of the Tenth International Driving Symposium on Human Factors in Driver Assessment, Training and Vehicle Design

\title{
INVESTIGATING PEDESTRIANS' CROSSING BEHAVIOUR DURING CAR DECELERATION USING WIRELESS HEAD MOUNTED DISPLAY: AN APPLICATION TOWARDS THE EVALUATION OF EHMI OF AUTOMATED VEHICLES
}

\author{
Yee Mun Lee, Jim Uttley, Albert Solernou, Oscar Giles, \\ Richard Romano, Gustav Markkula, Natasha Merat \\ Institute for Transport Studies, \\ University of Leeds, Leeds, UK \\ Email: y.m.lee@leeds.ac.uk
}

\begin{abstract}
Summary: This study investigated pedestrians' crossing behaviour in a virtual reality environment. One aim was to develop a framework for evaluating external Human-Machine Interfaces (eHMI) used by automated vehicles for future studies. Pedestrians were provided with a series of two approaching cars, which were travelling at either $25 \mathrm{mph}, 30 \mathrm{mph}$, or $35 \mathrm{mph}$, with eight manipulated time gaps in between cars, where the second car either decelerated or kept pace. These stimuli were presented in 3 blocks. Pedestrians' task was to cross (or not) naturally between the approaching cars. Data from decelerating trials were analysed. Results showed $51 \%$ of crossings happened before deceleration, $31 \%$ of crossings after the car had stopped and only $18 \%$ of the crossings during deceleration, leaving a great margin for evaluating the effect of eHMI and changing pedestrian crossing behaviour during deceleration. A learning effect was found, demonstrating a shift of decision making across blocks, whereby crossing increasingly occurred during the approaching vehicle's deceleration, rather than after it had come to a full stop. Further analyses were conducted to investigate the effect of speed on initiation time, crossing time and safety margin. This study provides guidelines in choosing the appropriate time gaps and speeds that may influence pedestrians' crossing decisions and behaviour while presenting different designs of eHMI in future studies. The results also provide guidance on how to evaluate safety, efficiency/receptivity and learning effects, when comparing different eHMI designs in VR experiments.
\end{abstract}

\section{INTRODUCTION}

Interactions between automated vehicles (AVs) and other road users are expected to increase in the future. Previous research has suggested the importance of external Human Machine Interfaces (eHMI), for communication of information by AVs (e.g. Merat et al., 2018; Deb et al., 2016; Fridman et al., 2017; Schieben et al., 2018). A number of methods have been used to study user experience and input in this context, for example video-based studies (Lagstrom \& Lundgren, 2015), survey studies (e.g. Deb et al., 2016, Fridman et al., 2017; Zhang et al., 2017), interviews (Clamann et al., 2016), and Wizard of Oz studies (Habibovic et al., 2018) evaluating the comprehension, trust, perceived safety, acceptance and preferences for such eHMI designs. It is useful to observe how different eHMIs affect pedestrians' actual crossing behavior in this context, although challenges exist regarding availability of real automated vehicles, and the ethics and safety of conducting such studies in the real world. Therefore, VR settings provide an ideal situation for studying this behavior. However, to our knowledge, there are a limited number of studies which have considered actual crossing behavior of pedestrians in VR. One such example is a recent study by Deb et al., (2018), where four auditory and four visual cues were used as eHMI, to notify pedestrians that it was safe to cross the road at an intersection. However, 
in this study, the vehicle always stopped at the intersection, which meant that it was always safe for participants to cross. Crossing time and waiting time were used to measure whether each eHMI was successful in influencing the participants' decision-making strategies. Compared to baseline, pedestrians only walked faster when a beep sound was provided, and slower when music and verbal sound was provided. We argue, however, that it is also important to investigate whether pedestrians are actually able to judge the approaching speed and deceleration behavior of vehicles during their crossing maneuver, in the absence of reassurance that the vehicle will always give way (see also Clamann et al., 2016). Any knowledge in this context will then help determine whether such judgement is enhanced by the presence of different eHMIs.

The aim of the current study was to use VR to contribute to a methodology and analysis framework for evaluating the safety, learnability and efficiency of future eHMI of AVs, by taking into account the approaching speed and deceleration profile (present, absent) of a vehicle during a pedestrian crossing task. In particular, we investigated whether pedestrians are able to use the deceleration profile of the vehicle, travelling at different speeds, when crossing the road. The current study adopted part of Lobjois and Cavallo's (2007; 2009) design and measures, such as their gap acceptance manipulations. Performance was measured in terms of the percentage of crossings made before the vehicle decelerated, during deceleration, and when the car had actually stopped. Initiation time, crossing time and safety margin were also analysed to better understand pedestrians' crossing behavior when the car was decelerating.

\section{METHOD}

\section{Participants}

Ethical approval was obtained from the University of Leeds Research Ethics Committee. (Ref: LTTRAN-097). Fourteen participants (9 Male), aged 19-61 $(\mathrm{M}=27.64$, S.D. $=10.57)$ took part in the study. To ensure a degree of homogeneity in behaviour, all participants were required to have been resident in the UK for at least a year, and did not suffer from any mobility impairments. The study took 90 minutes to complete, which included a short briefing session, completion of a consent form, a short practice session with the VR scenario and equipment, and completion of a series of questionnaires following the VR study. A Misery Rating (Bos et al., 2010) questionnaire, completed after each block, confirmed that participants did not feel nauseous. Participants were reimbursed $£ 15$ for taking part in the study.

\section{Apparatus}

The study was conducted in a spacious room at the University of Leeds (13m x 9m). Participants wore HTC VIVE head-mounted display (HMD) (Figure 1). Unity software was used to create the virtual environment; a handheld controller was used by participants to control the scenarios.
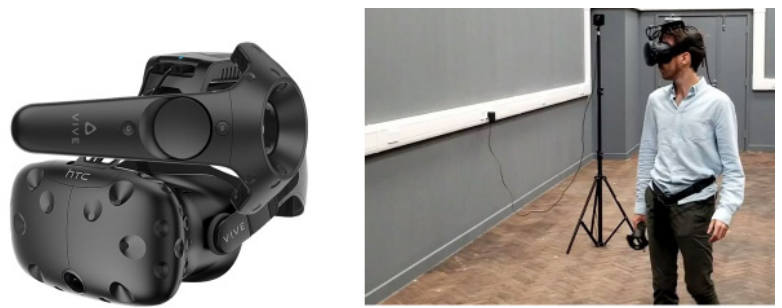

Figure 1. HTC VIVE head-mounted display and controller (Left) and the set up (Right)

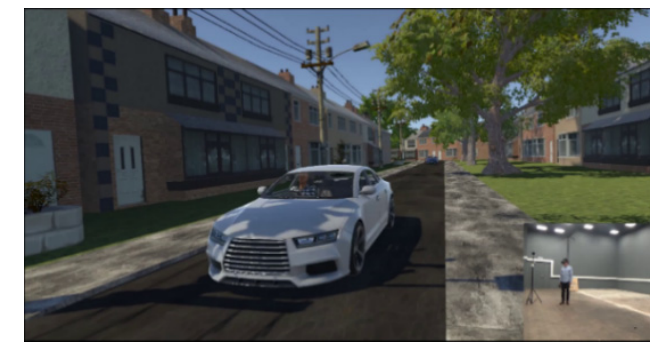

Figure 2. An example of the stimuli and the virtual environment. 
The virtual environment incorporated a 3.5 meter single lane road (UK standard) during daytime (see Figure 2). A row of trees was created on one side of the road to indicate the starting point of each trial. Two saloon vehicles (one white, one blue) approached from the participant's right. Two grey bollards showed the crossing path to guide pedestrians (see also Figure 3 ).

\section{Design and Procedure}

A within-subject design was used and 48 stimuli were created ( 3 approaching speeds, 8 time gaps, 2 deceleration profiles). Each trial consisted of two approaching cars (white followed by blue), which always travelled at the same speed (either $25 \mathrm{mph}, 30 \mathrm{mph}$ or $35 \mathrm{mph}$ ). A time gap of $1-8 \mathrm{~s}$ (in 1s increments) was introduced between the cars to provide variability. For each travelling speed/time gap combination, $50 \%$ of the following vehicles decelerated at 32.5 meters before the crossing path (Point A in Figure 3) stopping at point B (2.5 meters before reaching the participant). This created a fixed stopping distance of 30 meters. Therefore, the deceleration rate was $2.72 \mathrm{~m} / \mathrm{s}^{2}$ for a car that was travelling at $25 \mathrm{mph}, 3 \mathrm{~m} / \mathrm{s}^{2}$ for $30 \mathrm{mph}$, and $4.08 \mathrm{~m} / \mathrm{s}^{2}$ for $35 \mathrm{mph}$. This design was based on the typical stopping distance suggested in the UK Department for Transport's (2007), Rule 126.

The dependent variables included pedestrians' crossing decision and behavior (see more in results). For each crossing maneuver, participants began by standing at the edge of the road when they were ready. They were instructed to press the button on the controller to trigger the first trial. Two cars then approached from the right, and the pedestrian's task was to cross naturally, if they felt safe to do so, between the two approaching cars. After crossing the road, they had to walk back to the starting position, and press the button again to trigger the next trial. If they did not cross in that particular trial, they would have to press the button to trigger the next trial. The practice session stopped whenever participants felt they understood the experimental task (approximately after 5 minutes). Following the practice block, the first block of the experimental session (48 trials) then began in a random sequence, and the procedure was the same as the practice session. To investigate learning effects, a total of three blocks ( 3 x $48=144$ trials) was administered. To reduce fatigue effects, there was a short break between blocks.

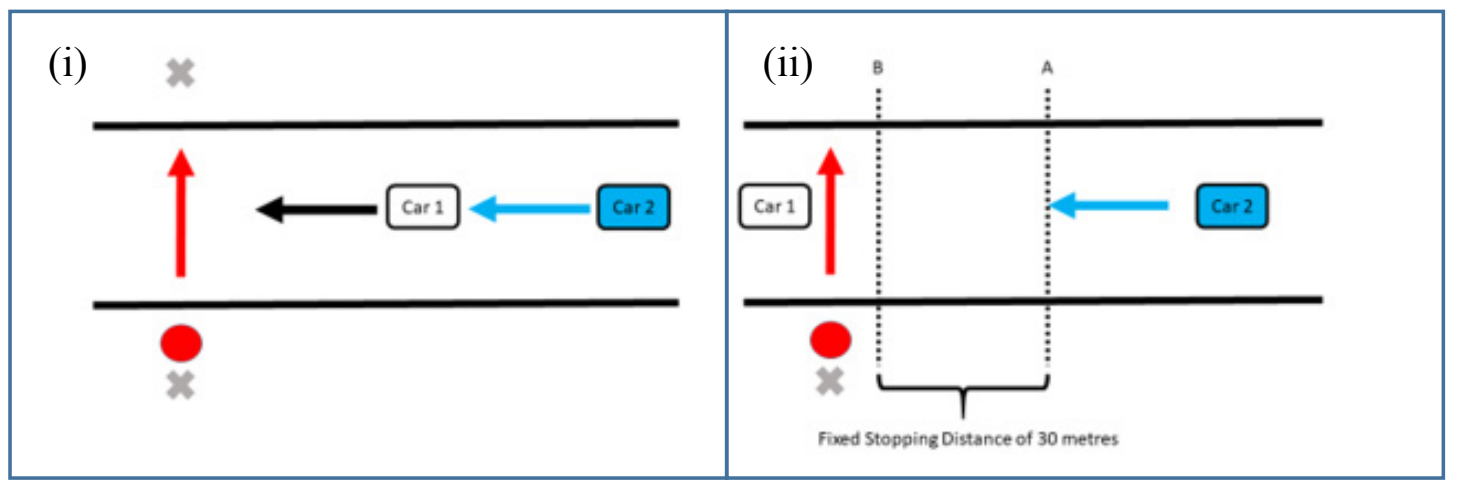

Figure 3. The left picture (i) shows the two approaching cars (white followed by blue) that were travelling from the right. Red dot represents the pedestrian who was standing at the edge of the road waiting to cross (direction shown by red arrow). The two grey crosses depict the grey bollards that were created to guide the participants' crossing path. The right picture (ii) shows that the first car has now passed the pedestrian, and the gap is now open for a pedestrian to cross. Point A shows where the second car would start to decelerate and stop at point $B$. 


\section{RESULTS AND DISCUSSION}

Crossing behavior for the deceleration trials (1008 trials) was analyzed, with an amalgamation of all time gap manipulations, as they were not the main interest and only used to provide variability. Data analysis was divided into three sections, based on vehicle behavior: i) before vehicle deceleration (before point $\mathrm{A}$ in Figure 3ii) during deceleration (between point A and B) and iii) after the vehicle had stopped (point B, see Figure 3ii). We investigated the percentages of crossings that were made in each of these sections. All participants' movements and positions were measured by the position of the HMD.

\section{Percentage of crossings}

Results showed that in 51\% of cases, pedestrians had crossed the road before the car started to decelerate, with $31 \%$ of crossings occurring only after the vehicle had stopped. More interestingly, only $18 \%$ of crossings occurred while the car was decelerating.

A 3 (25mph, 30mph, 35mph) x $3\left(1^{\text {st }}, 2^{\text {nd }}, 3^{\text {rd }}\right.$ block $)$ repeated measures ANOVA with Bonferroni post hoc correction was conducted to investigate the effect of speed and learning on crossing behavior. There was no interaction found in any of the analyses, and therefore figures below provide an overview of the main effect of speed (Figure 4) and learning (Figure 5) on crossing behavior.

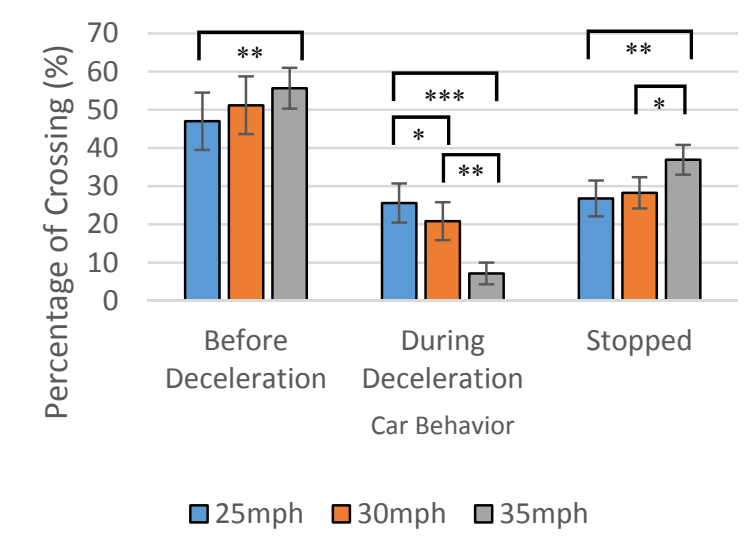

Figure 4. The effect of speed on the percentage of crossings. Error bars depict S.E.M. *** indicates $p$ $<.001 ; * *$ indicates $p<.01$, * indicates $p<.05$

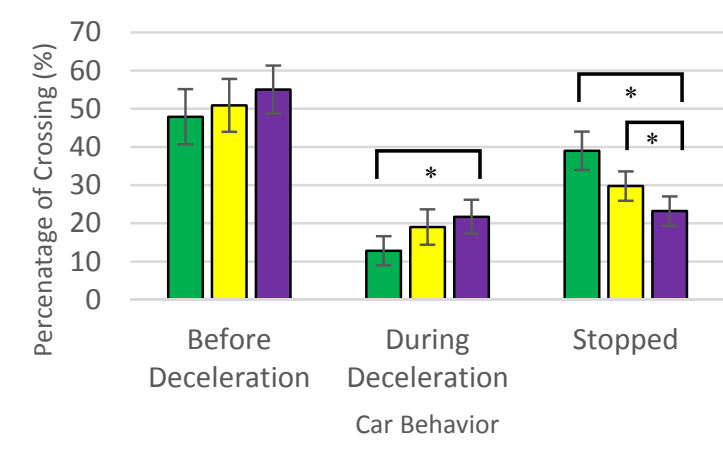

$\square$ Block $1 \square$ Block $2 \square$ Block 3

Figure 5. The effect of learning across 3 blocks on the percentage of crossings. Error bars depict S.E.M. *** indicates $p<.001 ; * *$ indicates $p<.01$, * indicates $p<.05$

Before Deceleration - There was a significant effect of speed on crossing behavior, $(F(2,26)=$ $10.48, p<.001, \eta^{2}=.446$ ), where a systematically lower number of crossings were made as speed decreased. This finding, which is in line with the results reported by Lobjois \& Cavallo (2009), is thought to be because higher travelling speeds provided larger distance gaps, for the same time gaps. There was no learning effect, whereby the same pattern was seen for crossing behavior before the deceleration of the vehicle, for all three blocks.

During Deceleration - A main effect of speed was also found during the deceleration phase $\left(F(2,26)=19.37, p<.001, \eta^{2}=.598\right)$, but the reverse trend was observed, where less crossings were made with higher travelling speed. This finding suggests that pedestrians hesitated when a car that travelled at a higher speed decided to decelerate, even though the higher deceleration rate 
should have been more noticeable. A main effect of block was also found for this condition $\left(F(2,26)=4.52, p=.021, \eta^{2}=.258\right)$, with a trend in more crossings observed across block.

Stopped Vehicle- A main effect of speed on crossing behavior was found $(F(2,26)=5.16, p=$ $\left..013, \eta^{2}=.284\right)$. The percentage of crossings was higher when the vehicles travelling at higher speeds came to a stop. A main effect of block was also found in this condition $(F(2,26)=4.33, p$ $=.024, \eta^{2}=.250$ ). The trend showed that the percentage of crossings decreased across blocks. As participants had learned the behavior of the vehicle by the third block, most had already crossed before the vehicle had come to a complete stop.

\section{Crossing Behaviour During Vehicle Deceleration}

Looking more specifically into the "during deceleration" phase, we examined the initiation time, crossing time and safety margin. The initiation time was the time taken for the pedestrian to make their first move after the rear end of the first car had crossed the crossing path. Crossing time was the time spent to cross the road, and safety margin was described as the time taken for the front of the second vehicle to arrive at the crossing path after the pedestrian completed the crossing (Lobjois \& Cavallo, 2007).

Initiation Time -A one-way ANOVA was conducted to compare the initiation time for the three speeds. Results revealed no main effect of speed, $(F(1,18)=0.84, p>.05)$ (see Figure 6).

Crossing Time -The main effect of speed was not found to affect crossing time $(F(1,18)=$ $0.84, p>.05)$. Pedestrians spent the same amount of time crossing the road for all 3 speed categories (see Figure 6).

Safety Margin-There was a main effect of speed, $(F(1,18)=16.69, p<.001)$, whereby safety margin was lower for higher speeds (see Figure 6). This result is expected since there was no difference in initiation time and crossing time across the three different speeds.

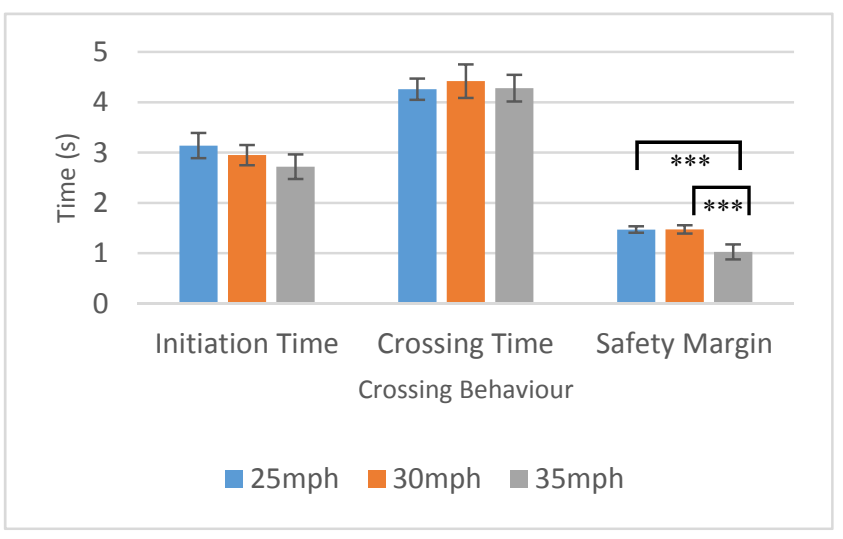

Figure 6. The effect of speed on pedestrians' initiation, crossing time and safety margin (s) during deceleration. Error bars depict S.E.M. *** indicates $p<.001$; ** indicates $p<.01$, * indicates $p<.05$

\section{SUMMARY AND CONCLUSIONS}

Results revealed that, when faced with approaching cars at different speeds, pedestrians crossed the road most often before the car started to decelerate. Only $18 \%$ of crossings were made when the car was decelerating. Initiation of this crossing behavior, and crossing time, were not found to be affected by the vehicle's approaching speed, but the safety margin adopted was lower for higher travelling speeds. Findings of this study provide some guidance in terms of the time gaps and speeds which could potentially change pedestrians' crossing decision and behaviour. Results showed that, after crossings that occurred before the vehicle decelerated (51\%), most crossings were achieved when the vehicle had stopped (31\%). This bimodal crossing pattern is in line with 
previous studies using other methods to investigate pedestrian crossing, such as simulation models (Markkula et al., 2018), or test track studies (Schneemann \& Gohl, 2016).

Considering the use of an eHMI in this context is therefore worth investigating, to establish whether such an interface can provide any further assistance to pedestrians interacting with AVs, especially in the absence of an active or responsible human operator. A shift of crossing decisions, and therefore a learning effect, was shown, demonstrating that pedestrians were more likely to cross early (more crossings before the decelerations, and less after the car had stopped) across the three blocks. This finding suggests that pedestrians learned about the deceleration of vehicles in the virtual environment, confirming the potential benefit of using such devices for investigating the value of different eHMI designs for research on AVs. Deb et al. (2018) suggest that a good eHMI design should provide a clear message at first exposure, maintaining the correct interpretation throughout. Regarding pedestrians' crossing behaviour, this study showed that an approaching vehicle with the highest speed produced the smallest safety margins (consistent with Lobjois \& Cavallo, 2007). Results also revealed that the crossing initiation time was the same for different speeds, creating a distance of $7.14 \mathrm{~m}, 5.48 \mathrm{~m}$ and $4.36 \mathrm{~m}$ for the vehicles travelling at 25,30 and $35 \mathrm{mph}$, respectively. This evaluation method could be applied to studying the efficiency in processing information provided by different eHMI designs, where better eHMI designs should show a lower initiation time, to indicate faster information processing and decision making by pedestrians, also providing a higher safety margin.

The findings of the current study provide guidance for evaluating the effectiveness of different eHMI designs, by using different objective measures, such as safety, efficiency/receptivity and learning effect, used for understanding the best means of communication of future AVs with other road users, in a mixed traffic urban environment. In future studies, we will be applying these objective measures to evaluate the effectiveness of eHMIs with these suggested criteria.

\section{REFERENCES}

Bos, J.E., de Vries, S.C., van Emmerik, M.L., \& Groen, E.L. (2010). The effect of internal and external fields of view on visually induced motion sickness. Applied Ergonomics, 41(4), 516521.

Clamann, M., Aubert, M., \& Cummings, M. L. (2017). Evaluation of Vehicle-to-Pedestrian Communication Displays for Autonomous Vehicles (No. 17-02119).

Department for Transport. (2018, June 27). General rules, techniques and advice for all drivers and riders (103 to 158). Retrieved November 16, 2018, from https://www.gov.uk/guidance/the-highway-code/general-rules-techniques-and-advice-for-alldrivers-and-riders-103-to-158

Deb, S., Carruth, D.W., Sween, R., \& Strawderman, L. (2017). Efficacy of virtual reality in pedestrian safety research. Applied Ergonomics, 65, 449-460.

Deb, S., Strawderman, L.J., \& Carruth, D.W. (2018). Investigating pedestrian suggestions for external features on fully autonomous vehicles: A virtual reality experiment. Transportation Research Part F, 59, 135-149. 
Deb, S., Warner, B., Poudel, S., \& Bhandari, S. (2016). Identification of external design preferences in autonomous vehicles. In: Proceedings of the 2016, industrial and systems engineering research conference. Anaheim, California.

Fridman, L., Mehler, B., Xia, L., Yang, Y., Facusse, L. Y., \& Reimer, B. (2017). To Walk or Not to Walk: Crowdsourced Assessment of External Vehicle-to-Pedestrian Displays. arXiv preprint arXiv:1707.02698.

Lagstrom, T., \& Lundgren, V. M. (2015). AVIP-Autonomous vehicles interaction with pedestrians [Doctoral dissertation, Thesis]

Lobjois, R., \& Cavallo, V. (2007). Age-related differences in street-crossing decisions: The effects of vehicle speed and time constraints on gap selection in an estimation task. Accident Analysis and Prevention, 39(5), 934-943.

Lobjois, R., \& Cavallo, V. (2009). The effects of aging on street-crossing behavior: From estimation to actual crossing. Accident Analysis and Prevention, 41(2), 259-267.

Markkula, G., Romano, R., Madigan, R., Fox, C. W., Giles, O. T., \& Merat, N. (2018). Models of Human Decision-Making as Tools for Estimating and Optimizing Impacts of Vehicle Automation, Transportation Research Record.

Merat N, Louw T, Madigan R, Wilbrink M, \& Schieben A. (2018). What externally presented information do VRUs require when interacting with fully Automated Road Transport Systems in shared space? Accident Analysis and Prevention, 118, 244-252.

Morrongiello, B.A., Corbett, M., Milanovic, M., Pyne, S., \& Vierich, R. (2015). Innovations in using virtual reality to study how children cross streets in traffic: evidence for evasive action skills. Injury Prevention, 21(4), 266-270

Schieben, A., Wilbrink, M., Kettwich, C., Madigan, R., Louw, T., \& Merat, N. (2018). Designing the interaction of automated vehicles with other traffic participants: design considerations based on human needs and expectations. Cognition, Technology and Work, $O(0), 0$.

Schneemann, F., \& Gohl, I. (2016) Analyzing Driver-Pedestrian Interaction at Crosswalk: A Contribution to Autonomous Driving in Urban Environments. Presented at IEEE Intelligent Vehicles Symposium.

Simpson, G., Johnston, L., \& Richardson, M. (2003). An investigation of road crossing in a virtual environment. Accident Analysis and Prevention, 35(5), 787-796.

Zhang, J., Vinkhuyzen, E., \& Cefkin, M. (2017). Evaluation of an autonomous vehicle external communication system concept: A survey study. In International conference on applied human factors and ergonomics, 650-661. 